\title{
Fuente y recorrido lingüístico de una retraducción en judeoespañol: el caso de La alma de hombre (Constantinopla, 1855)*
}

\author{
Doğa Filiz Subaşı** \\ Yozgat Bozok Üniversitesi \\ ORCID ID: https://orcid.org/0000-0001-6975-6197 \\ Tania M. ${ }^{\mathrm{a}}$ García Arévalo*** \\ Universidad de Granada \\ ORCID ID: https://orcid.org/0000-0002-4100-445X
}

Entre los siglos XIX y xx el Imperio otomano fue objetivo de diferentes estrategias de evangelización llevadas a cabo por misioneros protestantes llegados de Estados Unidos y Gran Bretaña. Una de las principales consistió en el desarrollo de obras filantrópicas como la publicación de textos en las diversas lenguas vernáculas usadas por los grupos étnico-religiosos del Imperio, entre ellos, el judeoespañol. El objetivo de estas páginas es el de presentar el estudio de una de estas obras, inédita hasta la fecha, $L a$ alma de hombre (Constantinopla, 1855), centrando nuestra atención en el recorrido lingüístico que sufre hasta llegar a la comunidad sefardí y la identificación de su fuente.

Palabras ClaVe: Imperio otomano; modernidad; misiones; cristianismo; sefardíes; judeoespañol.

Source and Linguistic Journey of a Retranslation into Judeo-Spanish: The Case of LA ALMA DE HOMBRE (Constantinople, 1855).- Between the $19^{\text {th }}$ and $20^{\text {th }}$ centuries, the Ottoman Empire was the target of different evangelization strategies carried out by Protestant missionaries from the United States and Great Britain. One of the main ones consisted of launching philanthropic works such as the publication of texts in the various vernacular languages used by the ethnic-religious groups of the empire, including JudeoSpanish. The objective of these pages is to present the study of one of these works,

* Estas páginas son resultado del proyecto The Almost-Extinct Sephardi Community in Ankara: A Cultural, Artistic and Linguistic Heritage from Past to Present (6602cFEN/19-262, 2019-2022) concedido por la Unidad de Coordinación de Proyectos de Investigación Científica de la Universidad de Yozgat Bozok (Turquía).

**doga.subasi@bozok.edu.tr

*** taniagarcia@ugr.es

Copyright: (C) 2021 CSIC. Este es un artículo de acceso abierto distribuido bajo los términos de la licencia de uso y distribución Creative Commons Reconocimiento 4.0 Internacional (CC BY 4.0). 
unpublished to date, La alma de hombre (Constantinople, 1855), shedding light on the linguistic journey that it undergoes until it reaches the Sephardic community and the identification of its source.

KeYwords: Ottoman Empire; Modern Age; Missions; Christianity; Sephardim; Judeo-Spanish.

\section{INTRODUCCIÓN}

Los siglos XIX y XX del Imperio otomano se caracterizan por ser un periodo convulso. Las circunstancias cambiantes que tuvieron lugar en el plano económico o político -la proclamación de las Tanzimat entre 1839 y 1876, la revocación de la dimma o la progresiva adquisición de derechos civiles, entre otras- afectaron a los grupos religiosos que habitaban en territorio del Imperio y supusieron la aparición de nuevos escenarios hasta ese momento desconocidos. Pese a ello, la comunidad judía sefardí otomana del siglo XIX vive un proceso de florecimiento y europeización que desemboca en una revitalización cultural y lingüística y, aunque la Alianza Israelita Universal (París, 1860) jugará un papel clave en esta transformación de carácter modernizador y educativo ${ }^{1}$, es importante resaltar que el contacto con Occidente ya se había dado de hecho, antes de que esta se instaurara en el Imperio: primero, en el siglo

${ }^{1}$ Sobre la actuación de la Alianza Israelita Universal, véanse Rena MoLHo, «The Moral Values of the Alliance Israelite Universelle and their Impact on the Jewish School World of Salonika and Morocco», El Prezente: Estudios sobre la cultura sefardi: La cultura Judeo-Espanyola del Norte de Marruecos 2 (2008) págs. 127-137; Aron Rodrigue, French Jews, Turkish Jews: The Alliance Israelite Universelle and the Politics of Jewish Schooling in Turkey 1860-1925 (Bloomington: Indiana University Press, 1990); Michael M. LASKIER, «Aspects of the Activities of the Alliance Israélite Universelle in the Jewish Communities of the Middle East and North Africa: 18601918», Modern Judaism 3:2 (1983) págs. 147-171; Joseph HACKER, «The Intellectual Activity of the Jews of the Ottoman Empire During the Sixteenth and Seventeenth Centuries» en Jewish Thought in the Seventeenth Century, eds. Isadore Twersky y Bernard Septimus (Cambridge, Mass.-London: Harvard University Press, 1987) págs. 96-135; Esther Benbassa, «The Process of Modernization of Eastern Sephardi Communities», en Sephardi and Middle Eastern Jewries. History and Culture in the Modern Era, ed. Harvey Goldberg (Bloomington: Indiana University Press, 1996) págs. 89-98, y Dina Moustani, (ed.) Jewish Education in Southeastern Europe (mid 19thmid 20th Century) (Volos, 2021 [en prensa]). 
XVI, con las misiones de los jesuitas ${ }^{2} \mathrm{y}$, más tarde, con las de los protestantes que buscaban la conversión de judíos, cristianos y musulmanes.

Entre 1820 y 1914 se observa un flujo constante de grupos protestantes que llegan a diferentes localizaciones del Imperio otomano venidos, sobre todo, de Estados Unidos como la American Board of the Commissioners for Foreign Missions o de Gran Bretaña con la London Missionary Society ${ }^{3}$ y los integrantes de la London Society for Promoting Christianity among the Jews Mission ${ }^{4}$ a los que, posteriormente, se unieron los de la Church of Scotland, la Iglesia Anglicana y la Free Church of Scotland ${ }^{5}$, estando la mayor parte del trabajo de estos últimos

2 Sobre las misiones jesuitas, véanse José Marín Riveros, «Alteridad e identidad. Los jesuitas en Constantinopla en el siglo XvIII», Byzantion Nea Hellás 17-18 (19981999) págs. 125-146, y Thomas Banchoff y José Casanova, (eds.), The Jesuits and Globalization: Historical Legacies and Contemporary Challenges (Washington DC: Georgetown University Press, 2016).

3 Fundada originalmente en 1795 como The Missionary Mission y con el objetivo general de dar a conocer el cristianismo a las naciones paganas, en 1818 pasará a denominarse The London Missionary Society. En torno a ella puede consultarse la obra de Bernard Thorogood, (ed.), Gales of Change: Responding to a Shifting Missionary Context: The Story of the London Missionary Society, 1945-1977 (Geneva: WCC Publications, 1994); Andrew PORTER, Religion versus Empire? British Protestant Missionaries and Overseas Expansion, 1700-1914 (Manchester: Manchester University Press, 2004). La School of Oriental and African Studies de la Universidad de Londres (SOAS) posee sobre ella una colección de documentos dentro del Council for World Mission Archive disponible en $<\mathrm{http} / / / \operatorname{archives.soas.~}$ ac.uk/CalmView/Record.aspx src $=$ CalmView.Catalog \&id $=$ CWM\&pos $=1>\quad y$ $<$ https://www.soas.ac.uk/library/archives/collections/missionary-collections/>.

${ }^{4}$ La London Society, también conocida como LJS, se enmarca en el movimiento judeocristiano que comenzó a principios del siglo XIX en Inglaterra y cuyos integrantes mostraban un perfil particular: judíos que se habían convertido al cristianismo o cristianos que trataban de presentar o simbolizar el cristianismo a los judíos. La fundación de la LJS en 1809 supuso un momento crucial en los inicios del movimiento asociado, en un primer momento, al converso de origen germano Joseph S. C. F. Frey; $c f$. Noam SiEnNA, "'It is a Minhag': Algerian Judaism Through the Eyes of a Hebrew-Christian Missionary», Journal of Jewish Thought 1:5 (2015) págs. 23-41: 24.

${ }^{5}$ Surgida como una escisión de la Church of Scotland en 1843 como protesta por la intromisión del estado británico en los asuntos de la iglesia. Véase Ryan MALlon, «A Church for Scotland? The Free Church and Scottish Nationalism after the Disruption», Scottish Church History 49:1 (2020) págs. 1-24. 
dirigido a judíos de origen askenazí e italiano ${ }^{6}$. No parece ser, sin embargo, que estos fueran los pioneros ya que, con anterioridad, en los siglos XVI y XVII, misioneros católicos franceses y británicos, sobre todo franciscanos, se habían instalado en tierras osmanlíes ${ }^{7}$, concentrando su

${ }^{6}$ Leah Bornstein-Makovetsky, «The Activity of the American Mission Among the Jews of Istanbul, Izmir, and Salonika during the Nineteenth Century», en Days of the Crescent. Chapters in the History of the Jews in the Ottoman Empire, ed. Minna Rozen (Tel Aviv: Tel Aviv University, 1996 [en hebreo]) págs. 273-310: 274-278. Aunque en estas páginas nos ocupamos del caso específico del Imperio otomano, hemos de señalar que el interés de las actividades protestantes era el de estar presente en todas las localizaciones judías que les fuera posible y, de esta manera, llegaron a puntos tan lejanos como, por ejemplo, Marruecos, Bombay, Pest o Ámsterdam. Con respecto a este hecho, véase Rachel (Saba) Wolfe, «From Protestant Missionaries to Jewish Educators: Children's Textbooks in Judeo-Spanish», Neue Romania 40 (2011) págs. 135-151: 136, nota 4, en la que menciona la obra de Eliezer Bashan, The Anglican Mission and the Moroccan Jewry in the Nineteenth Century (Ramat-Gan: Bar-llan University, 1999 [en hebreo]), pero también añadimos la de Jean-Louis Miege, «Les Missions Protestants au Maroc, 1875-1905», Hesperis 42 (1955) págs. 153-186; el artículo de Mitch NumArK, «Hebrew School in Nineteenth-Century Bombay: Protestant Missionaries, Cochin Jews, and the Hebraization of India's Bene Israel Community», Modern Asian Studies 46:6 (2012) págs. 1764-1808; el trabajo de Yaron Perry, British Mission to the Jews in Nineteenth-Century Palestine (London: Frank Cass, 2003); la tesis doctoral de Ábrahám Kovács, The History of the Free Church of Scotland's Mission to the Jews in Budapest and its Impact on the Hungarian Reformed Church, 1841-1914 (Universidad de Edimburgo, 2003, accesible en $<$ https://era.ed.ac.uk/handle/1842/30370?show=full >); los artículos de Jan MAES, «'Die Joden zijn bij mij per toeval gevonden', Hoe protestanten Joden redden in de omgeving van Antwerpen en Leuven (1942-1944)'», Les Cahiers de la Mémoire Contemporaine 10 (2011) págs. 271-317, y Jaap Cоцтноғ, «From Juvenile Criminal to Jewish Scholar: the Nineteenth-century Protestant Mission among Amsterdam Jews which Culminated in a Murder Attempt on a Clergyman», Jewish Historical Studies 48 (2016) págs. 138-157, así como la obra de carácter general de Irene Aue-Ben DAVID, Aya Elyada, Moshe Sluhovsky y Christian Wiese, (eds.), Jews and Protestants: From the Reformation to the Present (Berlin-Boston: De Gruyter, 2020).

7 Pueden verse: Elmira Vassileva, «Ottoman Istanbul from the Perspective of the Catholic Missionaries in the Post-Tridentine Period (End of 16th-17th centuries)», Osmanl İstanbulu 4 (2016) págs. 289-311 y, de la misma autora, «The activities of the Franciscan Order in the Ottoman territories (17th Century): Missionary approaches», Etudes balkaniques 3 (2017) págs. 415-449; Claude Prudhomme, "Centralité romaine et frontières missionnaires», Mélanges de l'école française de Rome 109:2 (1997) págs. 487-504; Adina Ruiu, «Conflicting Visions of the Jesuit Missions to the Ottoman Empire, 1609-1628», Journal of Jesuit Studies 1:2 (2014) págs. 260-280; Emese MunTÁN, «Uneasy Agents of Tridentine Reforms: Catholic Missionaries in Southern Ottoman Hungary and Their Local Competitors in the Early Seventeenth Century», Journal of Early Modern 
labor en ciertas áreas como la región siria y Egipto, así como en aquellas habitadas mayoritariamente por maronitas y coptos. A diferencia de estos, los americanos no limitaron su labor a zonas concretas y tampoco la redujeron a grupos étnico-religiosos determinados, sino que trataron de expandirla a todo el territorio ${ }^{8}$, no obstante, sí es reseñable que en el caso judío los mayores esfuerzos se focalizaran en tres enclaves: Esmirna, Constantinopla y, posteriormente, Salónica.

Las razones de estas campañas se movían entre razones religiosoideológicas, políticas y de índole económica, como apunta Martín González en un reciente estudio ${ }^{9}$ :

Protestants believed they were meant to accelerate the dawning of the millennium. Both the conversion of Jews and defeating the antiChrist were necessary to bring Jesus' Second Coming. All of these millennial ideas allowed Britain to regard itself as the New Jerusalem that was meant to re-Christianize the old Jerusalem and the Ottoman lands. Reasons for this colonialist agenda were not merely religious but they rather include a politicallor even an economic explanation. America also shared a common view, as they were thought to be the city upon a hill.

El propósito principal derivado de todas ellas era el de conseguir el mayor número de conversiones posible en una única dirección (judíoscristianos-musulmanes hacia el protestantismo) con el mínimo riesgo de retractación y, para lograrlo, se idearon varias estrategias estrechamente ligadas entre sí: evangelización a través de la prédica en público o en privado y la distribución de biblias, panfletos y otros tratados religiosos; obras filantrópicas como la creación de escuelas para posibilitar la es-

Christianity 7:1 (2020) págs. 151-175, y Paolo GIRARDELLI, «Between Rome and Istanbul: Architecture and Material Culture of a Franciscan Convent in the Ottoman Capital», Mediterranean Studies 19 (2010) págs. 162-188.

8 Devrim Üміт, «The American Protestant Missionary Network in Ottoman Turkey, 1876-1914», International Journal of Humanities and Social Science 4:6.1 (abril 2014, accesible en <http://www.ijhssnet.com/view.php?u=http://www.ijhssnet.com/journals/ Vol 4_No_6_1_April 2014/3.pdf\$) págs. 16-51: 17; también Robert M. HEALERS, «The Jews in Seventeenth Century Protestant Thought», Church History 46:1 (1979) págs. 63-79.

9 Daniel Martín-González, «An Ethnolinguistic Study of Sephardic Jews and their Language in the Ottoman Empire in the 19th Century in the Eyes of Scottish Protestant Missionaries», Darom. Revista de Estudios Judios 3 (2021) págs. 55-79: 57. 
colarización y educación de la población ${ }^{10}$; y las misiones médicas a través del establecimiento de hospitales ${ }^{11}$. A todos ellos, hay que añadir las humanitarias ${ }^{12}$, sobre todo, a partir del último cuarto del siglo xIx, y la prensa y publicaciones derivadas de esta - biblias, tratados religiosos, revistas, materiales de enseñanza para las escuelas ${ }^{13}$, entre otros- que algunos estudiosos consideran la cuarta y quinta rama, respectivamente, de las actividades de las misiones ${ }^{14}$.

${ }^{10}$ Es necesario mencionar que, aunque las escuelas no tuvieron éxito desde una perspectiva religiosa, sí lograron introducir la educación moderna en la sociedad sefardí en Constantinopla; $c f$. WoLfE, «From Protestant Missionaries to Jewish Educators», pág. 137, y Daniel Martín GonzÁlez, «Hidden vs. Overt Protestant Propaganda in an Educational Book in Judeo-Spanish: Alexander Thomson's Silabario (Constantinople, 1855)», Cadernos de Estudos Sefarditas 21 (2019) págs. 29-47: 31. Su popularidad, por otra parte, queda probada en extractos como el publicado por The Jewish Herald and Record of Christian Effort for the Spiritual Good of Ancient's People en 1850 en el que, dentro de su sección Corner for the Young People y auspiciada por la Free Church of Scotland, pone de relieve el trabajo que estos hacían en las escuelas femeninas de Constantinopla y la aceptación que estas tuvieron; cf. The Jewish Herald and Record of Christian Effort for the Spiritual Good of God's Ancient People (London: Aylott \& Jones, Paternoster Rows, 1850) vol. 5, pág. 108.

${ }^{11}$ Leah Bornstein-Makovetsky, «Activity of the American Mission among the Jews of Istanbul, Salonika and Izmir in the 19th century» en The Days of the Crescent: Chapters in the History of the Jews in the Ottoman Empire [en hebreo], ed. Minna Rozen (Tel Aviv: Tel Aviv University Press, 1996) págs. 273-310: 274-277; WoLfE, «From Protestant Missionaries to Jewish Educators», pág. 136; Joseph K. GreEne (Leavening the Levant [Boston: The Pilgrim Press, 1916] pág. 146) sostenía, en cuanto a la American Board y a sus obras médicas, que, en un principio, no estaban destinadas a la población sino a los misioneros y a sus familias: «Finally the American Board awoke to the necessity of sending medical missionaries, primarily for the sake of the missionary families, and, secondarily, for the general influence of medical practice among the people».

12 Destacamos el volumen colectivo de Inger Marie OKKenHaUg y Karène SAnCHEZ Summerer, (eds.), Christian Missions and Humanitarianism in The Middle East, 18501950 (Leiden: Brill, 2020).

13 Véase Nivi Gomel, Judeo-Spanish Hebrew Teaching Textbooks in the Ottoman Empire: Methods and Trends 1823-1935 (Tel Aviv: Bar-llan University, 2006).

14 Wolfe («From Protestant Missionaries to Jewish Educators», pág. 136) y Leah Bornstein-Makovetsky ( $\ll$ Jewish Conversion to Protestantism in the Ottoman Empire in the Communities of Istanbul and Izmir Until 1856», Miscellanea Historico-Iuridica 18 [2019] págs. 7-36: 8) señalan que las actividades se manifiestan en tres planos (evangelizador, educador y filántropo) en tanto que Üміт («The American Protestant Missionary», 
Esta labor de producción y publicación de obras que llevaron a cabo las misiones en el Imperio otomano se desarrolló teniendo en cuenta los factores lingüísticos que condicionaban su lectura por los diferentes grupos a los que trataban de evangelizar, esto es, llevando a cabo una intensa labor de traducción a las lenguas vernáculas presentes en el Imperio como el griego, el armenio, el armeno-turco, el árabe, el búlgaro y el judeoespañol ${ }^{15}$ al que usualmente se referían como hebreo-español ${ }^{16}$.

Dentro de la colección de publicaciones en judeoespañol se encuentra La alma de hombre, trabajo que vio la luz en Constantinopla en 1855 sin firma de autoría. Se conserva en la biblioteca del Instituto Ben-Zvi con el número 436 dentro de la colección de manuscritos y libros de Avner Perets ${ }^{17}$ y está digitalizado en la National Library de Israel dentro de la Rare Collection con la signatura $8=53$ A $399^{18}$, siendo este último el ejemplar base usado para nuestro análisis. Hasta la fecha ha permanecido inédito en su estudio y no se conocen acercamientos a su examen desde otras perspectivas a excepción de alguna mención aislada ${ }^{19}$.

El objetivo de estas páginas será el de ofrecer una aproximación a la caracterización de esta obra a través de dos factores: primero, la identificación de la fuente de la que deriva, el Child's Book on the Soul (1831),

págs. 17-18) sí diferencia y añade de forma independiente las acciones humanitarias y las editoriales.

15 Este hecho no es aislado, sino que se reprodujo de forma general en todos los lugares a los que llegaron las misiones, traduciendo los textos bíblicos a un total de 450 lenguas y dialectos; $c f$. William A. WONDERLY y Eugene A. NIDA, «Linguistics and Christian Missions-II», The Bible Translator 15:3 (1964) págs. 107-116: 107.

16 Son muy relevantes las impresiones y denominaciones que poseen los misioneros protestantes sobre la consideración del judeoespañol que se recogen en MARTíN-GonZÁLEZ, «An Ethnolinguistic Study of Sephardic Jews», págs. 68-72, en las que se lo califica como una jerga, un dialecto, una lengua, una mezcla de español y hebreo o una corrupción (págs. 69-70).

${ }^{17}$ El ejemplar del Instituto Ben-Zvi no se encuentra digitalizado pero puede consultarse su registro y características en su ficha disponible en $<\mathrm{http} / /$ folkmasa.org/cat $/ \mathrm{mb}$ _shirp4. php?mishtane $=436>$.

${ }^{18}$ La obra posee el número de sistema 990012024690205171 y se encuentra accesible en <https://www.nli.org.i1/en/books/NNL_ALEPH001202469/NLI>.

19 Aitor García Moreno, «Poemas castellanos en textos sefardíes: ejemplos en $\mathrm{La}$ escalera a la anyeźadura (Constantinopla 1853 y 1888)», Sefarad 78:1 (2018) págs. 149-200: 152 , nota 6. 
del misionero americano Rev. Thomas Hopkins Gallaudet y pionero en la educación a personas con dificultades auditivas en Estados Unidos; y segundo, la problemática en cuanto a su recorrido lingüístico -del original inglés, al búlgaro a través de Odesa hasta llegar a Esmirna y Constantinopla pasando por múltiples traducciones coetáneas a otras lenguas vernáculas hasta llegar al sefardí-. No nos ocuparemos en esta ocasión de las características de la lengua que exhibe y que, sin duda, merecen un estudio más exhaustivo.

2. Datos DE LA OBRA: Título, SubTítulo, LUGAR DE EDICIÓN, IMPRENTA Y AÑO

Al acceder al ejemplar de la National Library encontramos en su portada los elementos básicos: el título (La alma de hombre, en letra merubá ); un subtítulo (Para el amyeźamiento de las criaduras, en letra raši ); una cita bíblica extraída de Prov 1, 8 en merubá vocalizada (traducción: 'Escucha, hijo mío, la instrucción de tu padre y no abandones la enseñanza de tu madre' ${ }^{20}$ ); el lugar de edición (Constantinopla, en raší); la imprenta (A. Churchill, en raší) y el año de su publicación en calendario gregoriano y su correspondencia con el calendario judío (1855-5615). El volumen original del Ben-Zvi sí contiene el total (120 páginas) pero en el de la National Library se han perdido de 41 a 44, de 97 a 100 de forma parcial y de 101 a 120 de manera completa.

Como puede observarse, la autoría no consta, no obstante, a falta de más datos que puedan conducir nuestra investigación en una dirección, señalamos la apreciación de que el Rev. Alexander Thomson, misionero de la Free Church of Scotland, pudiera haber influido en su publicación tal y como apunta Aitor García Moreno ${ }^{21}$.

De todos los elementos anteriores, quizá el más relevante sea el de la imprenta por varias cuestiones. En primer lugar, el 1 de julio de 1846 la administración otomana reconocía a los protestantes como un millet, esto es, el reconocimiento oficial dentro del estado otomano de cada una

20 Puede verse Jesús Cantera Ortiz de Urbina «La sabiduría en el libro de los Proverbios del Antiguo Testamento», Paremia 16 (2007) págs. 19-27.

${ }^{21}$ García Moreno, «Poemas castellanos», pág. 152, nota 6. 
de las comunidades religiosas que les otorgaban autonomía, garantizándoles la continuidad religiosa y cultural permitiéndoles incorporarse al sistema político y económico ${ }^{22}$. Antes de esto, los misioneros americanos tuvieron dificultades para imprimir y publicar porque los patriarcas griegos y armenios se quejaban ante el gobierno otomano cada vez que aquellos escribían o traducían documentos religiosos a sus lenguas. Debido a esto, el gobierno permitió las imprentas privadas en Estambul en los inicios del siglo XIX e incluso ayudó a los misioneros ya que consideraron que los autores tenían derecho a publicar en sus propias imprentas en tanto que los materiales les pertenecieran ${ }^{23}$.

A lo largo de la década de 1850 , hubo principalmente tres instituciones de impresión en Estambul ${ }^{24}$. La primera era una editorial oficial del Imperio -Matba' a-i Amire, fundada 110 años atrás-, teniendo los misioneros acceso a ella; la segunda, la oficina turca de periódicos o Takvim$i$ Vekayi; y una tercera pertenecía a Churchill ${ }^{25}$, apellido de William Nosworthy Churchill (1796-1846) ${ }^{26}$, empresario británico y propietario

22 Alejandra Álvarez SuÁrez, «La organización de los no musulmanes en el Imperio Otomano: millet y taifa», Collectanea Christiana Orientalia 9 (2012) págs. 23-45: 24. También S. Abu Jaber Kamel, «The Millet System in the NineteenthCentury Ottoman Empire», The Muslim World 57:3 (1967) págs. 212-223; Fatih ÖzTÜRK, «The Ottoman Millet System», Güneydoğu Avrupa Araştırmaları Dergisi 16 (2014) págs. 71-86, y Emrah ŞAHiN, «Ottoman Institutions, Millet System: 1250 to 1920: Middle East», en Cultural Sociology of the Middle East, Asia, and Africa (2012) págs. 181-183.

${ }^{23}$ Emrah SAHIN, Errand into the East: A History of Evangelical American Protestant Missionaries and Their Missions to Ottoman Istanbul During The Nineteenth Century (Ankara: Bilkent University, TFM, 2004) pág. 47.

24 Véase Abraham YAARI, Hebrew Printing at Constantinople: Its History and Bibliography (Jerusalem: Magnes Press, 1967).

25 ŞAHiN, Errand into the East, pág. 47.

26 Tenemos noticia del suceso calificado como 'El affaire Churchill' que este protagonizó. Durante una cacería en Kadıköy, parece que William N. Churchill hirió al hijo de un funcionario otomano, siendo arrestado y golpeado. Akif Pasha, entonces secretario de Asuntos Exteriores, no liberó inmediatamente a Churchill, lo que causó el recrudecimiento de las relaciones entre las autoridades otomanas y Gran Bretaña temporalmente. En cuanto a este, véase la narración de AKiF PAŞA, Un diplomate ottoman en 1836 (affaire Churchill) (París: E. Leroux, 1892, trad. Arthur Alric) y Murat R. ŞiviloĞLu, The Emergence of Public Opinion: State and Society in the Late Ottoman Empire (Cambridge: Cambridge University Press, 2018) págs. 171-172. 
del periódico Ceride-i Havadis ${ }^{27}$ al que sucedió su hijo Alfred Black Churchill ${ }^{28}(1820 / 26-1870)$ a su muerte. La imprenta toma su nombre de este último, a partir de entonces.

No será La alma de hombre la única obra que se publicará en la editorial A. B. Churchill sino que también tenemos noticia de otras como $\mathrm{La}$ escalera a la anveźadura (1853) ${ }^{29}$; Ele Toledot Bené Yisrael en su edición de 1854 pero no en la de 1868 por Artin Minasian, 1868 ni tampoco la de 1886, que fue publicada en A. H. Boyaĝian ${ }^{30}$; Gan na úl o La dotrina $y$ declaración del capitulo 53 de Yeš ayá. Traśladado del inglés (1854) ${ }^{31}$; El catecismo menor (1854) ${ }^{32}$ o Toledot Yešú 'a haMašíah o Sesenta y nueve cuentos tirados del Nuevo Testamento $(1857)^{33}$, entre otras.

\section{TeXto fuente de LA ALMA DE HOMBRE}

La alma de hombre - a partir de ahora $L A H$ - no resulta un trabajo original sino que es una retraducción de la obra fuente The Child's Book on the Soul -a partir de ahora TCBS- publicado originalmente en 1831 en Estados Unidos (Hartford) por la editorial Cooke \& Co. cuyo autor fue el

27 Sobre este periódico, véase Orhan KoloĞLU, «Newspapers», en Encyclopaedia of the Ottoman Empire, eds. Gábor Ágoston y Bruce Masters (USA: Facts on File, 2009) págs. 431-434: 432 y algunos datos sobre Churchill en Jutta CIKAR, Türkischer Biographischer Index (Walter de Gruyter, 2011) pág. 235.

28 Johan StRAuSs, «The Greek Connection in Nineteenth-Century Ottoman Intellectual History», en Greece and the Balkans: Identities, Perceptions and Cultural Encounters, ed. Dimitri TsIovas (London-New York: Routledge, 2017) págs. 47-67: 51, y Gordon GoodwIN y Joseph CoонILL «Churchill, Alfred Black (1825-1870), journalist and publisher», en Oxford Dictionary of National Biography, accesible en <https://www.oxforddnb.com/ view/10.1093/ref:odnb/9780198614128.001.0001/odnb-9780198614128-e-5393>.

29 Estudiada en García Moreno, «Poemas castellanos en textos sefardíes».

30 Estudiada en Daniel MarTín GonZÁlez, «Convergencias y divergencias lingüísticas con el judeoespañol castizo de un traductor nativo de inglés. El caso del Ele Toledot Bené Yisrael (Constantinopla, 1854 y 1886) del Rev. Alexander Thomson», Sefarad 80:1 (2020) págs. 267-296.

31 García Moreno, «Poemas castellanos en textos sefardíes», pág. 152, nota 6.

32 Estudiado en Aitor García Moreno, «iAnte el primer diccionario monolingüe judeoespañol?», Sefarad 73:2 (2018) págs. 371-408.

33 García Moreno, «Poemas castellanos en textos sefardíes», pág. 152, nota 6. 
Rev. Thomas A. Gallaudet (1787-1851), fundador y director del Asylum for the Dead and Dumb y pionero en la educación de niños sordos en Estados Unidos ${ }^{34}$. Casi inmediatamente después vio la luz su segunda parte y, aunque hubo numerosas ediciones que sí las publicaron por separado, como las de 1832, en otras se decidió unirlas en un único volumen, como la de 1848 , compartiendo esta última la estructura de $L A H$. Debido a esta cuestión, se ha usado para el análisis la edición de 1848 .

$T C B S$ posee un objetivo principal que Gallaudet especifica en su prefacio $^{35}$ y es el de enseñar a los niños:

One simple truth, that a child has a soul, distinct from the body, which will survive it, and live for ever ${ }^{36}$, is all that is attempted to be illustrated and enforced,

haciéndoles sentir que:

$\mathrm{He}$ is not a mere animal; that he has other and higher enjoyments $[\ldots]$ that he is an intellectual, moral, and accountable being, destined to an endless existence beyond the grave; and you have laid a foundation for teaching him that there is a GOD, in whose hands is his eternal Destiny; and that there is a Book, in which he can learn all that it is important for him to know with regard to the will of God, and his own happiness and duty ${ }^{37}$.

Para ello, utiliza diferentes ejemplos que ilustran y refuerzan la idea de que «his body will die, and be laid in the grave and turn to dust; but his soul will never die, that it is immortal $\left.{ }^{38}\right\rangle$, dejando en las madres la

${ }^{34}$ Sobre Gallaudet, véanse Henry BARnARD, Tribute to Gallaudet - A Discourse in Commemoration of the Life, Character and Services, of the Rev. Thomas H. Gallaudet (Hartford: Brockett \& Hutchinson, 1852); Henry BARNARD, «Eulogy», American Annals of the Deaf and Dumb 4:2 (1852) págs. 81-136, y Edward Miner Gallaudet, Life of Thomas Hopkins Gallaudet. Founder of Dead-Mute Instruction in America (New York: Henry Colt and Comp, 1888).

35 Gallaudet desarrolla con mayor profundidad los criterios y métodos de enseñanza aplicados en este libro en «Philosophy of Language», American Annals of Education 1:8 (1831) págs. 70-77 y 117-123.

36 En este extracto y los siguientes se conservan las mayúsculas y cursivas originales utilizadas por Gallaudet para resaltar las ideas expuestas.

37 TCBS 1848, pág. vii.

38 TCBS 1848, pág. vi. 
responsabilidad de que instruyan a sus hijos en la enseñanza religiosa cristiana mediante la lectura de este libro ${ }^{39}$. En suma, su contenido proporciona todo un tratado de contenido filosófico que, en su segunda parte, adquiere un mayor peso teológico tratando exclusivamente sobre la caracterización del alma y de Dios.

La técnica educativa que utiliza para lograr los propósitos anteriores es la del diálogo. La obra está estructurada en torno a diferentes conversaciones entre una madre y un hijo sobre diversos temas, textos sobre los que seguidamente se plantea una batería de preguntas para que los pequeños reflexionen sobre las lecturas ${ }^{40}$. En tanto que la fuente original posee 24 diálogos (dialogues) -divididos en 12 la primera parte y 12 en la segunda-, la retraducción judeoespañola presenta una primera parte (págs. 1-50) en la que se conservan 12 hablas -págs. 41 a 44, las coincidentes con la habla 11 están perdidas- y una segunda parte (págs. 51-120) en la que se conservan únicamente 8 hablas $^{41}$. Las intervenciones de los protagonistas en las conversaciones en TCBS se hacen utilizando la inicial del nombre de cada uno, a excepción de la primera vez -en la que sí se señala el nombre completo-, y esto se plasma también en la $L A H$.

Si en TCBS los protagonistas son una madre, Mrs. Stanhope, y su hijo Robert de cinco años, en $L A H$ lo serán el padre, Ya'acob, y su hijo Re'ubén. En ambos el otro progenitor ha fallecido y el que queda a cargo de la familia decide llevar la instrucción de los hijos en casa. Se encuentra asimismo una segunda hija de tres años, de nombre Eliza $(T C B S)$ y Sará $(L A H)$ que no toma parte en los diálogos, pero cuya figura sí sirve para ejemplificar las enseñanzas. Este hecho es compartido con otros personajes a los que solo se menciona como Uncle John (TCBS) o Abraham (LAH); William Baker (TCBS) o Šemuel $(L A H)$ y Aunt Mary $(T C B S)$ o Malká ( $L A H$, sobrina y no tía de Re'ubén como en $T C B S$ ), por tanto, en la traducción judeoespañola se hebraízan los nombres para adaptarlos a los potenciales lectores de la comunidad judía sin provocar extrañeza entre ellos.

39 TCBS 1848, pág. vii.

40 En las ediciones de 1832 las preguntas sobre los diálogos se recogen al final de cada una de las partes o de los libros; sin embargo, en la de 1848, estas aparecen tras cada diálogo, coincidiendo con la estructura de la retraducción judeoespañola.

41 El ejemplar del Instituto Ben-Zvi conserva, como en el texto base, las 12 hablas en la segunda parte. 


\subsection{Comparaciones textuales texto fuente y texto meta}

Con el objetivo de mostrar la cercanía que la retraducción judeoespañola comparte con el original inglés, veamos varios ejemplos en el primer diálogo (Dialogue I / Habla $1^{42}$ ) que, a su vez, sirve como introducción para ubicar la escena, los personajes y el contexto en el que se desarrolla:

ROBERT STANHOPE was five years old. His father died when Robert was a little boy. His mother has one other child, Eliza, who was three years old. They lived in a pleasant town, in a small white house, near to the church, and to the school-house. Robert and Eliza did not go to school. Their mother said, they should go when they were a little older. She used to teach them at home. She was a very kind mother, and they both loved her very much. Behind Mrs. Stanhope's house there was a beautiful garden. One day she was walking in it with Robert. He picked some pretty flowers, to give to his little sister who was in the house, and tied them together with a string which he has in his pocket. After they had walked some time, they sat down on a seat, under a large, shady tree. It was in the afternoon, just before sunset. They breathed the pure, refreshing air. They smelled the sweet flowers which grew around them. They listened to the songs of the birds in the branches over their heads. It was calm and pleasant. Robert had been a good boy, and he felt very happy. Mrs. Stanhope felt happy too. It always made her happy to see Robert a good boy. She took hold of his hand and kissed him. She thought, she would walk a little with him, and teach him some good things. So she began.

Re'ubén tenía cinco años. Su mađre murió cuando él era muy chico. Tenía y una hermana de tres años que se llamaba Sará. No se habían iđo a la escola, siendo señor Ya'acob, su pađre, quis̀o amveźarlos en caśa. Él era buen pađre y ellos lo amaban mucho. Detrás de la caśa había una güerta hermośa. Un día, señor Ya'acob se estaba paseando allí con su hijo Re'ubén. Arancó algunas flores para dar a la hermanica que estaba en caśa. Las ató con un peđacico de hilo. Después de pasearse un poco, se asentaron debajo de un árbol. Se hiźo tarde, cerca encerarse el sol. Se alevantó un aire fresco. Olieron buenos güeśmos de las flores que crecieron en la güerta. Sintieron las cantigas de las aves que estaban ariba en los árboles. Re'ubén, siendo buen hijo, se alegró; el pađre también se gustó de ver el hijo alegre. Pensó de hablar

42 TCBS 1848, pág. 13; LAH 1855, pág. 1. 
con él un poco para amỵeźarle algunas buenas cośas de castiguerio. (Ansí empezó)

Tras esto comienza el diálogo en el que se tratan conceptos como la diferencia entre seres con vida (una rosa), seres sin vida (una piedra) o más abstractos como el tiempo (encarnado en un reloj) que corre, pero no tiene vida ${ }^{43}$ :

TCBS:

R. No, mother; a stone cannot speak.

Mrs. S. If you should try to teach it, could it learn any thing?

R. No, no, mother; you know it could not.

Mrs. S. Look at the beautiful rose in the nosegay which you have picked for Eliza. It is very different from the pebble. It has a stem and green leaves. It has soft, red and white leaves; and all put together, so as to make a very pretty flower. When it was on the rose-bush, it lived and it grew. And it will live a day or two longer, if Eliza puts it into a tumbler, and fills it with water. The rose is much more curious than the pebble. It lives, but the pebble does not. Talk to the rose, and see, if it will answer you.

R. Mother, it will do no good for me to talk to the rose. Rosses cannot hear or speak.

\section{$L A H$ :}

R. No, mi señor. La pieđra no pueđe hablar.

P. Si querías amṿeźarla, ¿pueđe amṿeźarse?

R. Mi señor sabe que la pieđra no pueđe amỵeźarse.

P. Mira aquella hermośa rośa que arancaste por tu hermana. No es como la pieđra. La rośa tiene palicos y hoj́as verdes. Tiene también hoj́as blancas como velluđo: unas blancas, otras colorađas. Es una flor muy hermośa. Mientres que estaba en la raíź, tenía viđa y crecía. Y turará dos o tres días si Sará la meterá en un vaśo de agua. La pieđra no es como la rośa. La rośa tiene viđa, ma la pieđra no tiene viđa. Habla con la rośa, y mira si te dará repuesta.

R. No vale nađa para hablar con la rośa. Ni oye, ni habla.

43 TCBS 1848, págs. 14-15; LAH 1855, pág. 2. 
A lo largo de las dos obras, tanto en el texto base como en el meta y con la intención de que los lectores infantiles hagan un ejercicio de reflexión en torno al texto, se plasman las cuestiones referentes al texto. Pese a que los diálogos sean prácticamente idénticos en $T C B S$ y $L A H$, en las cuestiones finales sí parece que $L A H$ los presenta mucho más breves, eliminando algunas preguntas que se repiten o que inciden en aspectos que ya han sido contestados ${ }^{44}$ :

\section{QUESTIONS ON DIALOGUE I.}

Can a stone speak, or learn any thing? Can a lump of earth? Can a piece of iron? Tell me other things, like stone and iron, that cannot speak, or learn any thing. How is a rose different from a Pebble? What other things, besides a rose, live and grow? Can a rose speak, or learn any thing? Can a Pink? Can a tulip? Tell me other things, like a rose, that cannot speak, or learn any thing. What is inside of a watch? Is a watch alive? Does it move of itself? What is there inside of a watch, that makes the wheels go? What must be done, to make a watch go? Which is the most curious, the watch, or the rose? Can a watch speak, or learn any thing? Tell me some other things, that are curious, like a watch. Can they speak, or learn any thing? Tell me some other curious things, that cannot speak or learn any thing? Why cannot the Pebble, the rose, and the watch, speak?

\section{PREGUNTAS.}

¿Pueđe hablar o amỵeźarse la pieđra? ¿Pueđe hablar o amỵeźarse un peđacico de tiera o de fiero? Dime otras cośas que no pueđen hablar o amỵeźarse. ¿Qué es la diferencia entre la rośa y la pieđra? ¿Dime otras cośas que tienen viđa y crecen, ma no pueđen hablar. ¿Qué cośa hay ađientro la hora? ¿Camina la hora de sí miśma? ¿Qué haće rođear los caros? ¿Qué es de menester de haćer para que camine la hora? ¿Cuál es la cośa más compuesta, la hora o la rośa? ¿Pueđe hablar o amỵeźarse la hora? Dime otras cośas compuestas como la hora que no pueđen hablar ni amỵeźarse.

Al final de la primera parte, Gallaudet en TCBS incluye una carta en la que señala la intención y separa sus dos secciones: el libro original y la segunda parte que se hizo de él. Este hecho aparece también recogido en $L A H$ (págs. 49-50) como una nota del autor. Dada la si-

${ }^{44}$ TCBS 1848, págs. 16-17; LAH 1855, págs. 4-5. 
militud que ambas mantienen, recogemos aquí la retraducción judeoespañola:

Mis queriđos niños -meldađores de estas hablas-, ¡escuchađ!

Creo que habéš entendiđo tođo lo que habe escrito. Y creo que no lo olviđaréš nunca. Veš que Re'ubén quis̀o amỵeźarse mucho. Vośotros también debéš que ser alegres cuando vuestros pađres o vuestros hermanos más grandes, o vuestros amỵeźađores queren hablar con vośotros tocante a buenas cośas para dar comiđa a vuestras almas. Escuchađlos. Vos acordađ lo que vos dićen y los agrađeceđ por sus buenas veluntađes.

Debemos aprovecharnos de cađa libro que meldamos. Pođéš recibir bien de este librico, si vos acordáš que cađa uno de vośotros, tanto como Re’ubén, tiene la alma que yivirá después de morir, que turará de siempre ¡que nunca, nunca morirá!

Pensađ de este particular. Preguntađ a vuestros pađres y amỵeźađores tocante a la alma, y qué es lo que debéš de haćer para que vuestra alma sea buena y alegre después de morir.

Pueđe ser yo también vos diré más de vuestra alma en un otro librico como este.

Vuestro amigo

El escribiđor.

Como puede apreciarse, $L A H$ es prácticamente una retraducción literal que recoge de manera fiel el texto base. En la comparación de ambos no hay apenas desviaciones salvo en la cuestión de los nombres o la concreción en las preguntas finales que sí encontramos en el judeoespañol. No obstante, la obra merece un estudio profundo en cuanto a su registro lingüístico, a los detalles mínimos en los que pueda alejarse del original, su recepción dentro de las escuelas de las misiones entre los escolares o el uso que estos hicieron de él y una comparación con otros trabajos coetáneos.

4. Del original inglÉS AL JUDEOESPAÑOL: PROPUESTA DEL RECORRIDO LINGÜÍSTICO DE TCBS HASTA $L A H$

La obra de Gallaudet no fue traducida del inglés original al judeoespañol directamente, sino que presenta un recorrido lingüístico mucho 
más complejo en el que Elías Riggs (1810-1901) ${ }^{45}$, un misionero americano de la American Board of Commissioners for Foreign Missions, tuvo un protagonismo absoluto.

Elías Riggs, traductor y lingüista, estuvo desde muy joven dedicado al estudio de las lenguas como el griego y el hebreo, asistiendo al Amherst College primero y al Andover Theological Seminary después. En 1832, último año de sus estudios en este seminario, ayudó a Moses Stuart, profesor de literatura sagrada y padre de la ciencia bíblica en América, con las pruebas de algunas de sus publicaciones ${ }^{46}$. Tras graduarse, emprendió su misión con la American Board en Grecia (18321838) donde toma su primer contacto con la obra de Gallaudet, del que traduce al griego su Psychology, entre otros trabajos de distintos autores americanos, imprimiendo los ejemplares en su propia imprenta, una de las primeras del país ${ }^{47}$.

Suponemos que, dentro del conjunto de trabajos que tradujeron y publicaron en Grecia se encontraba TCBS por dos cuestiones: el trabajo original había visto la luz en 1831 cuando Riggs aún estaba en Estados Unidos y porque, tras el traslado de la prensa de la misión americana de Grecia a Esmirna en diciembre de 1833, aparecieron las primeras traducciones al griego moderno de esta obra ${ }^{48}$.

Tras su estancia en Grecia, Riggs es trasladado junto con su familia a Esmirna donde se ocupa de la imprenta y las labores editoriales traduciendo, preparando y publicando biblias, tratados, libros religiosos y

45 Sobre Riggs, véanse Henry O. DwIGHT, «A Mighty Worker Before the Lord», The Missionary Herald (1901) págs. 98-103; Charles Trowbridge Riggs, «Elias Riggs - A Pioneer in Turkey: Missionary of the American Board, from 1832 to 1901», The Missionary Review of the World 56:1 (1933) págs. 30-32, y Edward RIGGS, «Elias Riggs, the Veteran Missionary to Turkey», The Missionary Review of the World 14:4 (1901) págs. 267-271.

46 Mehmet Ali DoĞAn, «Elias Riggs and the Missionary Activities of the American Board of Commissioners for Foreign Missions (ABCFM) in Greece (1832-1838)», International Review of Turkology 4:8 (2011) págs. 5-22: 6-7.

${ }^{47}$ Leo Wiener, «America's Share in the Regeneration of Bulgaria», Modern Languages Notes 13:2 (1898) págs. 33-41: 33.

48 Véase el catálogo de publicaciones dentro de Rufus Anderson, History of the missions of the American Board of Commissioners for Foreign Missions to the Oriental Churches (Boston: Congregational Pub. Society, 1872) pág. 505. 
libros escolares en lenguas vernáculas ${ }^{49}$. En este momento, la financiación para las publicaciones de la American Board of Commissioners for Foreign Missions en Esmirna era gracias a los libros que se vendían de forma local y los fondos recaudados en Estados Unidos por sociedades como la American Bible Society, The British and Foreign Bible Society y The American Tract Society ${ }^{50}$. Sobre una de ellas, The British and Foreign Bible Society, tenemos conocimiento de que un agente suyo contactó con Riggs para que le enviara ejemplares de algunas de las publicaciones que habían llevado a cabo en Grecia con el objetivo de trasladarlas a Odessa y ser traducidas al búlgaro. Este hecho es narrado por el mismo Riggs en un texto fechado el 3 de octubre de 1841 en The Missionary Herald de ese mismo año ${ }^{51}$ :

October 3 - Mr. B. Barker, agent of the British and Foreign Bible Society, called us to ask for some specimens of our Greek publications, which he proposed to send to Odessa, with reference to their being translated into Bulgarian. I gave him the Life of David, the Child's Book on the Soul, and the tract on self-examination. Should the translations be executed so as to meet the approbation of the competent judges, they

49 Mehmet Ali Doğan, American Board of Commissioners for Foreign Missions (ABCFM) And "Nominal Christians": Elias Riggs (1810-1901) and American Missionary Activities in the Ottoman Empire (Utah: The University Utah, Tesis Doctoral, 2013) pág. 95.

50 DoĞAn, American Board of Commissioners for Foreign, pág. 96; sobre estas organizaciones, véanse Edward Caldwell Moore, «Bible Societies and Missions: Their Joint Contribution to Race Development», The Journal of Race Development 7:1 (1916) págs. 47-73; Richard ClOGG, «The Publication and Distribution of Karamanli Texts by the British and Foreign Bible Society Before 1850, I», The Journal of Ecclesiastical History 19:1 (1968) págs. 57-81 y «The Publication and Distribution of Karamanli Texts by the British and Foreign Bible Society Before 1850, II», The Journal of Ecclesiastical History 19:2 (1968) págs. 171-193; John FeA, The Bible Cause: A History of the American Bible Society (Oxford: Oxford Scholarship Online, 2016); Sonia HAZARD, «The American Tract Society and the Refinement of the Evangelical Book, 1825-1861», The Papers of the Bibliographical Society of America 114:2 (June 2020) págs. 127-194, y Elizabeth Twaddell, «The American Tract Society, 1814-1860», Church History 15:2 (1946) págs. 116-132.

51 Elías RigGs, carta del texto del 3 de octubre dentro del apartado «Smyrna» en The Missionary Herald: For the year 1841, vol. 37 de The Missionary Herald, publicado por la American Board of Commissioners for Foreign Missions por la editorial Crocker \& Brewsler de Boston pág. 287. 
can then be printed here, under our inspection at the same press. Mr. Barker recently employed to print the Bulgarian New Testament. A new and very interesting field of foreign distribution may thus be opened to the American Tract Society. Mr. Barker has travelled in Bulgaria and the neighboring regions, and both from personal observation and from correspondence, has long been convinced that it is a very inviting field for missionary effort - Mr. Riggs.

Al parecer, Barker envió las obras que le había entregado Riggs a un escocés que hizo que algunos estudiantes búlgaros las tradujeran y volvió a mandarlas a Esmirna para que fueran impresas. Aquí, Riggs empleó a Fontinov, un búlgaro, para revisar junto con él algunas de ellas -publicadas un año más tarde- y otras, como la primera parte del TCBS, fueron llevadas por Riggs a Constantinopla para que las repasara Orgianovich, un serbio que había establecido una imprenta nativa y se había convertido en un ardiente bulgarófilo ${ }^{52}$. De esta manera, en 1844 fue publicado el primer libro de $T C B S$ en búlgaro, con un total de 61 páginas y una tirada de 2000 copias $^{53}$. Esta fue la manera en la que TCBS llegó a Constantinopla, en búlgaro a partir del inglés, once años antes de que su versión judeoespañola viera la luz.

Otras lenguas vernáculas fueron empleadas en la traducción de $T C B S$ en tierras otomanas, como el armeno-turco ${ }^{54}$, sin embargo, en los registros e informes de la American Board of Commissioners no aparece la $L A H$ entre las traducciones al hebreo o al judeoespañol ${ }^{55}$. Por este hecho consideramos que su publicación podría haber visto la luz a través de la Free Church of Scotland cuyos misioneros compartían con los americanos la producción en la imprenta de A. B. Churchill. Esta afirmación supondría que la obra de Gallaudet hubiera pasado de los americanos a los británicos antes de que la misión de los primeros en Constantinopla finalizara y el único nexo entre ambos grupos, en un mismo territorio,

52 WIENER, «America's Share in the Regeneration of Bulgaria», pág. 77.

53 Véase en el catálogo de AnDERson, History of the Missions of the American Board, pág. 512.

54 19th Annual Report of the American Tract Society, 1833-1838 (Boston: Perkins \& Marvin) pág. 62 en la que se apunta que en 1855 se estaba haciendo la traducción al armenio en Constantinopla.

55 Véase Anderson, History of the missions of the American, págs. 513-514. 
tiempo y dedicación, fue la figura de otro misionero: William G. Schauffler $(1798-1883)^{56}$.

William G. Schauffler fue un misionero de origen alemán de la American Board con el que Riggs, mucho antes de coincidir en la misión otomana, mantenía una buena amistad. Se habían conocido años atrás en el Andover Theological Seminary y ambos eran miembros de la Society of Brethren, lo que favoreció que Riggs ayudara a Schauffler con su griego en tanto que este lo hiciera con el estudio del alemán ${ }^{57}$. Por otra parte, en tanto que la American Board había enviado a varios misioneros para operar entre los armenios y griegos de Estambul entre 1830 y 1850, Schauffler fue el único designado específicamente para los judíos y, lejos de llevar a cabo sus propias iniciativas, decidió cooperar con los miembros de la Free Church of Scotland, entre los que se encontraba Alexander Thomson (1820-1899). Además, era conocedor del judeoespañol ya que en 1849 finalizó la traducción de la Biblia al judeoalemán y al judeoespañol ${ }^{58}$.

Las buenas relaciones entre ambos pueden verse igualmente en las palabras que Thomson le dedica en un reporte oficial sobre la necesidad de incorporar obras de la Calw Book Society a la misión para conseguir su financiación: «Our friend, Mr. Schauffler, has kindly offered his services to make the requisite inquiries» ${ }^{59}$ o cómo Schauffler se refiere a Thomson:

... when Mr. (now Dr.) A. Thomson came, and there was no more doubt of the readiness and intention of our Scotch brethren to occupy the Sefardee field, I saw that Constantinople was lost to us ${ }^{60}$.

Como se comentó anteriormente en cuanto al problema de la autoría de $L A H$, parece ser posible que Alexander Thomson hubiera influido en

56 William G. Schauffler, Autobiography of William G. Schauffler: for Forty-Nine Years a Missionary in the Orient (New York: A.D.F. Randolph, 1887).

57 DoĞAN, «Elias Riggs and the Missionary Activities», págs. 7-8.

58 ŞAнin, Errand into the East, págs. 51-52.

59 The Home and Foreign Record of the Free Church of Scotland, vol. 4, (Edinburg-London: J. Nichol-J. Nisbet \& Co. August 1853-July 1854), pág. 16.

${ }^{60}$ Martín GonzÁlez, «Hidden vs. Overt Protestant», pág. 33 en referencia a Edwards Amasa Park, Schauffler, Autobiography of William. G. Schauffler, pág. 197. 
su redacción en tanto que era el encargado de la misión en ese momento, aunque no hemos encontrado constancia documental de este hecho en los informes consultados de la Free Church. Sin embargo, en una de sus obras, el Silaちario o ayuđa para amveźar a meldar para el uśo de las escolas de los judiós sefaradim (Constantinopla, 1855), un pequeño libro de 20 páginas cuyo objetivo era el de enseñar a los niños de las escolas a leer judeoespañol aljamiado ${ }^{61}$, sí recoge un ejemplo sobre el alma, en términos genéricos, que recuerda, en esencia, al contenido derivado de TCBS y $L A H$ en cuanto a que la persona muere pero el alma permanece y es eterna: La al-ma es-pi-ri-tu es, no se ve (SIL1855: 5) ${ }^{62}$.

De una forma $\mathrm{u}$ otra y no poseyendo certeza en cuanto al verdadero traductor de $L A H$, sí consideramos sin embargo que sin las figuras de Elías Riggs y William Schauffler no hubiera sido factible la publicación de $L A H$ en judeoespañol por el recorrido lingüístico que sufre la obra original de Gallaudet desde 1831 hasta 1855.

\section{Conclusión}

Pese a las dificultades que entraña la identificación de las fuentes de cualquier obra, se ha conseguido caracterizar aquella de la que deriva directamente La alma de hombre, siendo una retraducción del original inglés de Thomas A. Gallaudet The Child's Book on the Soul. Una complicación añadida a este proceso surge con varios factores; primero, con el recorrido lingüístico de la segunda durante veinticuatro años (1831 a 1855) a través de las lenguas (del inglés al búlgaro y de este a numerosas lenguas vernáculas del Imperio otomano, entre ellas el judeoespañol); segundo, el paso de la obra entre misiones (de la American Board of Commissioners y la de la Free Church of Scotland) e incluso, una tercera circunstancia, entre misioneros (Elías Riggs, William G. Schauffler o Alexander Thomson, todos traductores y lingüistas).

No obstante, y aun teniendo en cuenta los avances, el texto merece un estudio mayor que comprenda perspectivas de análisis lingüístico y

${ }^{61}$ Martín González, «Hidden vs. Overt Protestant Propaganda», pág. 36.

62 Martín GonzÁlez, «Hidden vs. Overt Protestant Propaganda», págs. 36-37. 
la observación de estrategias de traducción o retraducción empleadas por el autor o autores que formaron parte del proceso. Junto con ello, sería relevante la elaboración de una comparativa del resultado obtenido en este estudio con otros similares de obras coetáneas a ella ${ }^{63}$.

Recibido: $17 / 10 / 2021$

Aceptado: 29/10/2021

${ }^{63}$ En este sentido, se sugiere Daniel Martín-GonzÁlez, «Retranslation and Power: Attempts of Conversion of Sephardic Jews in the Ottoman Empire in the 19th Century by Scottish Protestant Missionaries through Retranslations from English Texts», en Translation and power, eds. Lucyna Harmon y Dorota OsuchowsKa (Berlin-New York: Peter Lang, 2020) págs. 69-77. 